\title{
ENCOUNTING FOR INTRA/INTERLAMINAR COUPLING BY USING BOTH IN-PLANE AND OUT-OF-PLANE STRAINS IN AN HYBRID INTERFACE MODEL
}

\author{
Gilles Lubineau* and Ping Hu \\ ${ }^{1}$ Physical Science and Engineering Division, COHMAS Laboratory, \\ King Abdullah University of Science and Technology (KAUST) \\ 23955-6900, Thuwal, Arabie Saoudite \\ * gilles.lubineau@kaust.edu.sa
}

The degradation modes of laminated composites have been studied for a long time. It is usual to distinguish between so-called "intralaminar" degradation mechanisms such as for example transverse cracks [1], and "interlaminar" mechanisms such as local or global delamination. One of the most difficult tasks is to accurately predict how these different mechanisms interact with each other. A well-known example is precisely the coupling between intra- and inter-laminar damage which significantly influences the mechanical response of laminates. These coupling mechanisms can be harmful (for example when transverse cracking accelerates degradation by local delamination of the interface) or beneficial (when transverse cracking activates new modes of interfacial dissipation, and in particular large-scale bridging mechanisms [2]).

The objective of this presentation is to pave the way for a pragmatic and efficient modeling of these effects. For this, we first contribute to the enrichment of experimental knowledge on this point, by the systematic study of the evolution of the bridging of the interlaminar interface as a function of the density of intralaminar cracks. Original tests are developed, for which cross-ply composites are first pre-cracked under plane loading in order to introduce a pre-defined crack density. These sequences are then subjected to macroscopic delamination tests, in order to evaluate the effect of this pre-cracking on the interface tenacity. Secondly, a modeling approach is proposed, based on a new type of interface model [3]. Unlike the classical cohesive elements, which only consider the out-of-plane part of the displacement jump at the interface, our hybrid model also uses the planar part of the displacement jumps. This planar part can be used to predict the level of cracking of adjacent folds and thus modify the behavior of the interface accordingly. This hybrid model is therefore local, easily implementable, and takes advantage of the large amount of work carried out in micromechanics in prediction of transverse cracking.

\section{References}

[1] P. Ladevèze and G. Lubineau. On a damage mesomodel for laminates: micro-meso relationships, possibilities and limits. Composites Science and Technology, 61(15):2149 $2158,2001$.

[2] P. Hu, D. Pulungan, R. Tao, and G. Lubineau. An experimental study on the influence of intralaminar damage on interlaminar delamination properties of laminated composites. Composites Part A: Applied Science and Manufacturing, 131:105783, 2020.

[3] P. Hu and G. Lubineau. An enriched cohesive law using plane-part of interfacial strains to model intra/inter laminar coupling in laminated composites. Composites Science and Technology, 200:108460, 2020. 